\title{
1 OmicsDB::Pathogens - A database for exploring 2 functional networks of plant pathogens
}

3 Bjoern Oest Hansen 1,2,3 Stefan Olsson 1,4

$4{ }^{1}$ State Key Laboratory for Ecological Pest Control of Fujian and Taiwan Crops, College of

5 Plant Protection, Fujian Agriculture and Forestry University, Fuzhou 350002, China.

62 OmicsDriven, Toelloese, Denmark

$7{ }^{3}$ Max Planck Institute of Molecular Plant Physiology, Am Muehlenberg 1, 14476 Potsdam,

8 Germany

$9{ }^{4}$ Plant Immunity Center, Haixia Institute of Science and Technology, College of Life Science,

10 Fujian Agriculture and Forestry University, Fuzhou 350002, China.

12 Abstract

13 Plant pathogens are a great threat to food security. To combat them we need an

14 understanding of how they work. Integrating large-scale omics datasets such as genomes

15 and transcriptomes has been shown to provide deeper insights into many aspects of

16 molecular biology. For a better understanding of plant pathogens, we aim to construct a

17 platform for accessing genomic and gene co-expression networks for a range of pathogens

18 and reference species. Currently we have integrated genomic and transcriptomics data from

1910 species (Fusarium graminearum, Ustilago maydis, Blumeria graminis, Neurospora

20 crassa, Schizosaccharomyces pombe, Saccharomyces cerevisiae, Escherichia coli,

21 Arabidopsis thaliana, Mus musculus and Homo sapiens).

22 Here we introduce OmicsDB::Pathogens (http://pathogens.omicsdb.org), a publicly available

23 web portal with an underlying database containing genomic, and transcriptomic data and

24 analysis tools. It allows non-bioinformaticians to browse genomic data and inspect and

25 compare biological networks across species. 
26 The information is modelled in a graph-based database, enabling flexibility for querying and

27 future extensions. Tools such as BLAST and Cytoscape.js are available together with the

28 option of performing GO enrichment analysis. The database also enables the user to browse

29 information such as Orthologs, Protein domains and publications citing a given gene.

30 Herein we describe how to use this platform for generating hypotheses for the function of a 31 gene.

\section{Availability and Implementation}

34 Currently, Omicsdb supports networks for 10 organisms and is freely available for public use at http://pathogens.omicsdb.org

\section{Introduction}

38 Plant pathogens are a great threat to the ever-growing population and the following need for food. To combat them, an understanding of their mode of action and of how the pathogenic processes work is essential. To be able to obtain useful knowledge from genomes, it is necessary to be able to correctly assign functions to gene products. Manual annotation is not a feasible option to annotate the ever-growing number of available genomes. Scientists, therefore, must rely more and more on predictions.

44 In-silico methods have gained popularity for automated annotation of gene functions, though

45 for it to be useful, it is important that the accuracy of the predictions, and number of genes

46 that can be annotated is high.

47 The guilt by association hypothesis is based on the observation that genes that participate in

48 similar biological processes or are involved in similar regulatory pathways tend to display

49 similar expression profiles (Wolfe et al., 2005). An increasingly popular method to utilize this

50 observation is using gene co-expression networks. Networks are a convenient way of

51 representing biological data and allow for the utilization of graph theory. By analysing the

52 structure of the networks, it is possible to identify communities within the network, where the

53 genes are more closely related to each other than to the rest of the network and are

54 enriched for specific biological processes.

55 The two main applications for co-expression network analysis are 1) To use a bait-gene from

56 a known pathway or with a known function, as a query, and identify possible genes within

57 the same pathway or with a similar function (Itkin et al., 2013) and 2) to suggest the

58 biological process or pathways given gene is involved in, based on the functions of its

59 neighborhood. With the increase in available biological networks, an increasingly important 
60

usage is the identification of common network patterns across species, this allows for more reliable transfer of knowledge from and across model species. Inter-network comparisons serve multiple purposes, they can improve identification of functionally related orthologs across species (Hansen et al., 2014), and aid in the identification of conserved subgraphs within or across species (Stuart et al., 2003).

The amount of data a researcher working within molecular biology needs to handle has grown exponentially during later years. Not just does that put a heavy load on the IT infrastructure, but also on the researcher that might not be a bioinformatics specialist. Handling and processing omics data are often a requirement these days, to be able to perform analysis on this kind of data, researchers need to be familiar with scripting languages such as Python or $\mathrm{R}$.

Curated databases have shown to be powerful tools for integration and analysis of data. This has led to an emergence of integrated web-based databases, changing the analysis of networks from being a task for specialist bioinformaticians, into a simple routine task for experimental molecular biologists, investigating specific genes or conditions. However, most of these databases focus on the major model species, such as Maize (Andorf et al., 2016), Budding yeast (Kim et al., 2014) or fission yeast (Vo et al., 2016). There exist very few options for researchers working on non-model species. Plant pathogens have until now had little representation in these databases. Electronic resources for Fusarium graminearum eFG (Liu et al., 2013) a model species in plant pathology (Zhang et al., 2019), contains functional annotation as well as annotations of transcription factors and curated and predicted pathogenic genes for fusarium. PHI-base (Urban et al., 2017) is a great resource for curated Pathogen-Host Interactions but is manually curated and thus limited in size. PhytoPath (Pedro et al., 2016), is a resource for genomic information related to plant pathogens. Other databases with microbial data that cover mainly model species are also available (Kim et al., 2014, 2015; Oughtred et al., 2019)

To tackle this problem, we here present OmicsDB::Pathogens, an integrated database of networks from 8 species: 6 model species (Escherichia coli, Saccharomyces cerevisiae, Saccharomyces pombe, Arabidopsis thaliana, Mus musculus, Homo sapiens), and 4 plant pathogens (Fusarium graminearum, Ustilago maydis, Blumeria graminis, Neurospora crassa and Magnaporthe oryzae), as well as orthologs from 158 other fungal pathogens, to ease the knowledge transfer to and from these. 
96 OmicsDB:: Pathogens is a database developed to assist experimental molecular biologists in 97 accessing and analyzing omics data.

98 The database provides intuitive navigation, allowing the researchers to store, browse,

99 analyse and compare their data, as well as handling metadata of both samples and

100 workflows. Another important use of the database is that it allows comparisons between

101 species that can allow the experimental researcher not just to infer possible functions of

102 genes not previously studied experimentally in detail. It also makes it possible to rank genes

103 of interest to study enabling more focussed research in sorting out gene function. We

104 provide an extensive amount of already processed publicly available genomic and

105 transcriptomic data.

106 Results and discussion

107 OmicsDB is a database that includes high throughput experimental data and information for

108 multiple species. Since we store data from biological networks, it was decided to go for the

109 graph-based database Neo4J for storing most of the data. Neo4J has previously been

110 evaluated by Have and Jensen for several graph processing problems related to

111 bioinformatics and compared with PostgreSQL, they found Neo4J to be faster in many

112 cases, though they also conclude that graph-based databases are not necessarily the best

113 choice for all problems (Have and Jensen, 2013). Neo4J uses the property graph model,

114 which means that nodes and edges can have key/value properties associated. Neo4J uses

115 its own query language, Cypher for querying the graph, cypher allows queries to be

116 formulated in terms of paths, which allows them to be concise and intuitive compared with

117 equivalent SQL queries, which is often complicated by joins, and difficult to read. Due to the

118 simplicity of traversing edges, and accessing data through the Cypher language, we decided

119 to use Neo4J for all data gene expression data, that is stored in an SQLite database, and flat

120 files that are stored in an S3 compatible object storage, with their paths and metadata stored

121 in Neo4J linked to the relevant organism. A simplified overview of our Neo4J data model can

122 be seen in Fig. 1 An overview of the infrastructure and data processing steps can be seen in

123 Fig. 2. The processed expression data is stored in a SQL database, with common identifiers

124 shared between the other systems. 

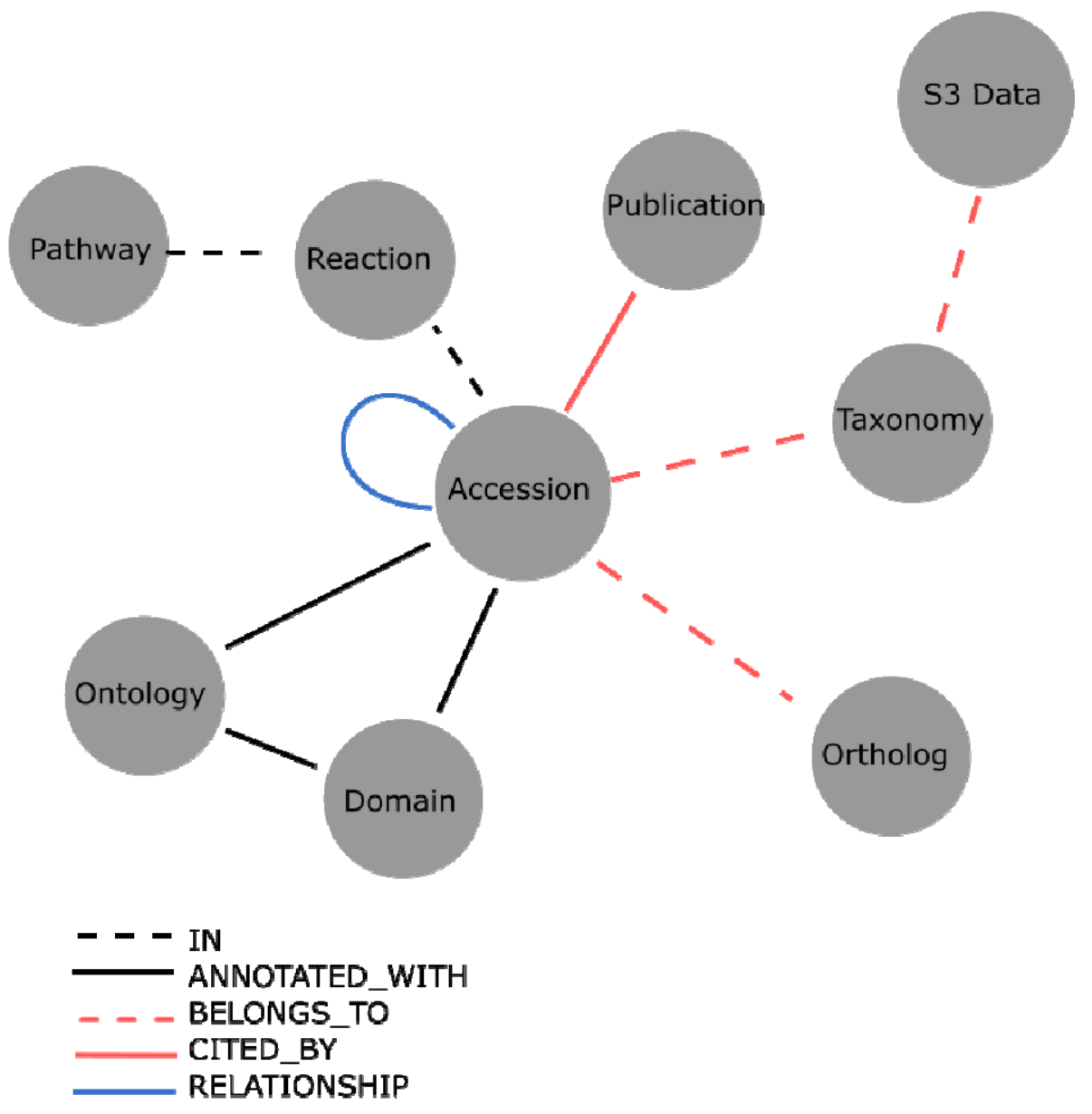

126 Fig 1. The part of our Neo4J data model. This shows how the different nodes are related.

127 Properties are left out for simplicity. Gathering data across any node is easy, for example, if

128 one wishes to know all publications related to a given Domain its possible to traverse the

129 graph from Domain over Accession to Publication. We believe this gives a more intuitive way

130 of accessing data than through a classical relational database.

131 FASTA and GFF files, are stored using an S3 compatible object storage (Palankar et al., 132 2008), together with their respective indexes. Accessing this information is easy since we 133 can traverse the graph from a given Accession to the S3 data, and use the Accession 134 together with the file index to drag out only what we need from the flat-file, while benefiting 135 from the features of S3 buckets, especially scalability and accessibility across servers.

136 To ease access to our data, we developed a web-based interface for OmicsDB::Pathogens 137 (http://pathogens.omicsdb.org). An overview of the website is shown in Fig 3. The site is 138 centered around gene information pages, where each page tries to summarize the 139 knowledge about a given gene, Users can search for the genes of interest using keywords, 140 gene identifiers or by performing a BLAST search against their sequence of interest. 


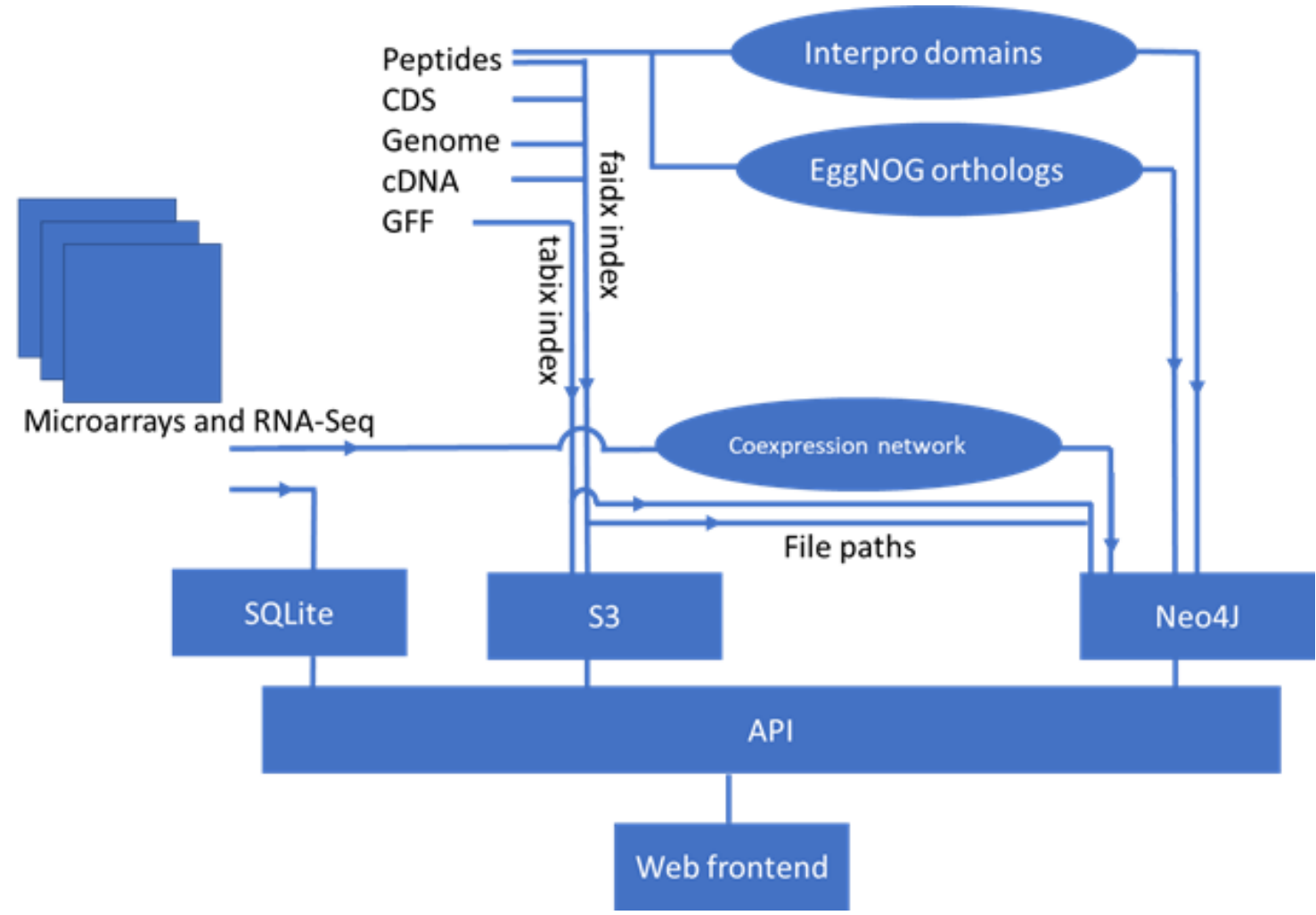

142 Figure 2. Storage and pipelines included in the data processing and storage. Microarray and

143 RNA-seq data are stored processed and normalized (not shown). The TPMs are then stored

144 in an SQLite database. The TPMs is also processed to generate a co-expression network,

145 stored in Neo4J. Peptide, CDS, cDNA, Genomes and GFF files are stored in an S3

146 compatible object storage, together with their respective faidx and tabix indexes (Li et al.,

147 2009; Li, 2011). Peptide sequences are processed by eggNOG (Huerta-Cepas et al., 2016)

148 to identify ortholog families, and by InterProScan to find conserved domains. Both orthologs

149 and domains are stored in Neo4J, connected to their respective peptides.

150 Basic functions of OmicsDB::Pathogens

151 The main source of information is the gene page that offers important information regarding

152 each gene. It provides different information for each gene. An overview can be seen in Fig 3.

153 The top of the page (Fig 3A) shows the basic information: The gene model with intron and

154 exon information. Gene Ontology annotations (Ashburner et al., 2000). Ortholog groups from

155 EggNOG (Huerta-Cepas et al., 2016) and protein domains from InterProScan (Hunter et al.,

156 2009; Jones et al., 2014) and the coding DNA sequence and the protein sequence. Further

157 down the page (Fig 3B) is a list of co-expressed genes and a visualization of the co-

158 expression network. As well as A bar chart of gene expression displaying the average of

159 replicates for a given experiment (Fig 3C). 
A)

\section{FGSG_03777}

\section{Gene info GFF Features}

Interpro: IPR012135 CS

EggNog ortholog group: COG0167eNOG

Uniprot I1RTYC

EC:1 (Oxidoreductases)

Location vs coexpression plot

160

161

162

163

164

165

166

167

168

169

170

171

172

173

174

175
$60-0006222$
60.0005207
60.00041se
$60-0004152$

\begin{abstract}
IIA
isa

IEA
\end{abstract}
UMP biosynthetic process $[5$
'de novo' wyimidine nucleobase biosynthetic process 6
dihydroerotate oxidase activity 5
dihydroeretate dehydrogenase activity 6

B)
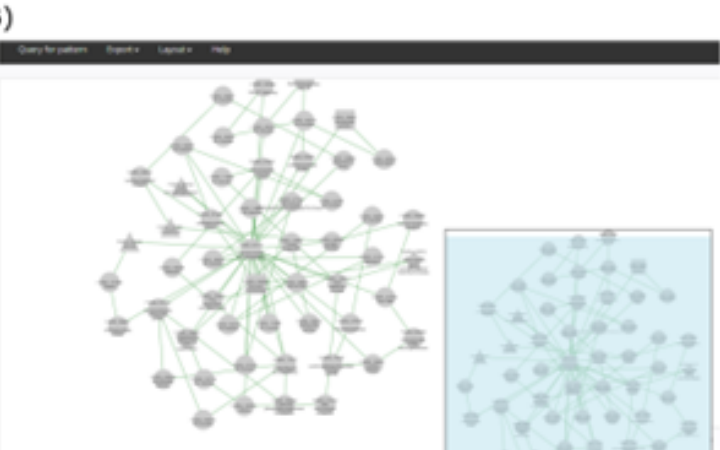

C)

Fig 3. An example of the gene profile page. A) Shows the structural and functional information on the gene as well as ortholog information, possible pathways, and InterPro domains. B) Shows an example of a co-expression network. C) Shows the expression profile of a given gene. Both $A, B$ and $C$ are found on the same page in the browser.

This page also serves as a gateway to explore the information within and across species.

For the species without networks, this data is limited to protein domains, ortholog families, DNA and protein sequences.

The network representation of gene interactions allows the users to query for the pattern of co-regulated orthologs across other species, to see if this regulated "module" is preserved. This function can ease the annotation transfer of "biological process" terms, relying not only on sequence similarity but also on the biological context.

To give a quick overview of what types of proteins the genes in the network code for, shapes of the nodes are used. The default shape is round, but it will be given a different shape in the case of transcription factors, or if a protein is classified with one of the 1-6 Enzyme 
176 commission classifications (Cornish-Bowden, 2014). The node representing the bait gene is

177 displayed in red. An overview of the shapes can be found in Supplementary Table 1. To

178 represent different types of relationships, the edges are colored using the following scheme,

179 Green edges: co-expression, red edges: physical interaction, blue edges: genetic interaction.

180 The physical and genetic interactions are from The BioGrid database (Oughtred et al.,

181 2019), the co-expression data is calculated using the Mutual Rank (MR) method (Obayashi

182 et al., 2009) modified to 1/MR with a MR threshold of 50.

184 From the gene page is it possible to access other pages with information. Ortholog pages:

185 Each EggNOG ortholog family has a dedicated page, with aggregated information on this

186 family. Including genes and species in the family, functional annotations as well as papers

187 citing the family. This enables users to easily check at a glance what information is available

188 from other species. As well as how diverse the family is by visualizing the distribution of

189 members per species in a pie chart. An example of an ortholog page can be seen in Fig 4.

190 Domain pages are similar to ortholog pages, but centred around a domain, for example,

191 PF00331 or PS51760.

A)

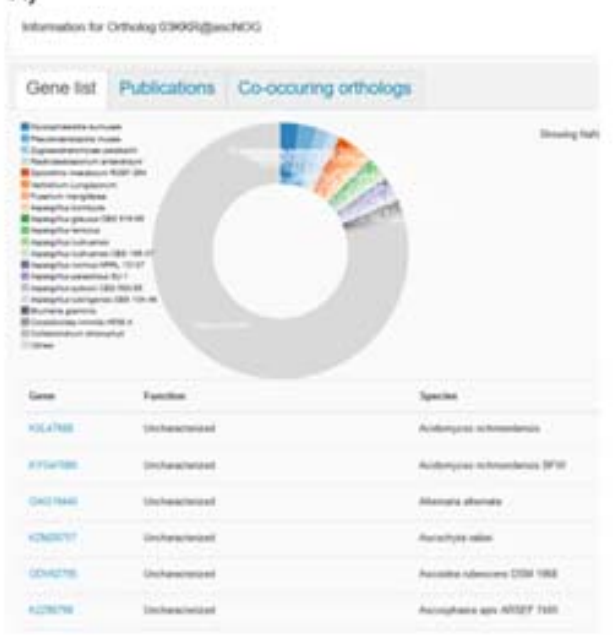

B)

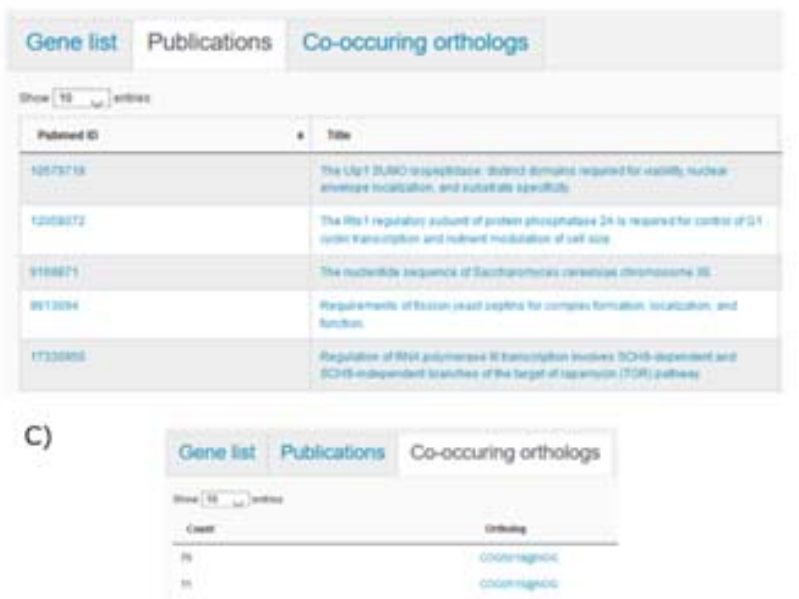

Fig 4. Each Ortholog has a unique page with multiple tabs. A) it shows all that has been assigned and the species they belong to. It is possible to sub select species using the pie chart. The other tabs include B) publications citing genes within this ortholog group. As well as C) orthologs that co-occur across the networks. Orthologs that often co-occur hints that they might participate in a similar process. 
201 those affiliated with a certain ontology in another species. Traditionally the user would have

202 had to download and annotate both datasets. However here it can be done with the click of a 203 button.

\section{Advanced functions}

205 OmicsDB also provides some tools enabling users to perform more advanced analyses. One

206 example is cross-species network alignment. Cross-species comparisons of biological

207 networks with interactions are still an emerging field, It allows for finding network patterns

208 that between orthologous genes across species, hinting towards similar function. This has

209 been used successfully in plants (Usadel et al., 2009; Movahedi et al., 2012; Hansen et al.,

210 2014). Most work on gene function prediction using gene co-expression so far has been

211 working on single or few species. Identifying conserved co-expression patterns across

212 orthologs in different species can identify highly relevant candidate genes sharing similar

213 functions or participate in the same pathway (Movahedi et al., 2012; Hansen et al., 2014).

214 An example of such a strategy with experimental confirmation is the study by ltkin et al.,

215 where comparative co-expression for tomato and potato was utilized, leading to the

216 discovery of a gene cluster that is related to the steroidal glycoalkaloids pathway (Itkin et al.,

217 2013).

218 It is also possible to use the surrounding network and look for enriched GO terms, using a

219 hypergeometric test. This is done at the bulk analysis page. Where a list of genes can be

220 analysed. A plot of the expression values for all genes in the list will be generated as well as

221 the GO enrichment. It is also possible to export both GO enrichments as well as peptide and

222 CDS sequences in fasta format.

\section{Use case: Vacuolar iron uptake}

224 Yeast Fet3/Ftr1 (YMR056W/YER145C) are genes for two proteins that are in yeast high-

225 affinity iron uptake at the plasma membrane (Askwith et al., 1994, 3; Stearman et al., 1996).

226 The proteins physically connect to each other. Ftr1 is a transporter and Fet3 is an

227 oxidoreductase. A similar system Fet5/Fth1 (YFL071W/YBR207W) exists at the vacuolar

228 membrane and is involved in regulating vacuolar iron storage (Urbanowski and Piper, 1999)

229 In Fusarium graminearum FGSG_02143 is known to be the plasma membrane Ftr1-like

230 protein and the homologous protein FGSG_05160 is probably the Fth1-like protein located at

231 the vacuole membrane. To test if this can be the case FGSG_05160 was used as bait and 
232 the result is displayed together with the networks for yeast Ftr1 and Fth1. The aligned

233 network can be seen in Fig. 5. Red edges represent possible orthologs and black edges co-

234 expression within the species. The subcellular location for some genes products in Yeast

235 has been marked.

236 What can be seen is that the vacuolar and the plasma-membrane iron transport is more co-

237 regulated in the $F$. graminearum data than in the yeast data (direct strong co-regulation

238 between FGSG_05160 and FGSG_02143). The pattern of co-regulation of the other proteins

239 with orthologues in yeast also supports that FGSG_05160 is a vacuolar located Fth1-

240 orthologue. This has, however, to be confirmed by direct experiments.

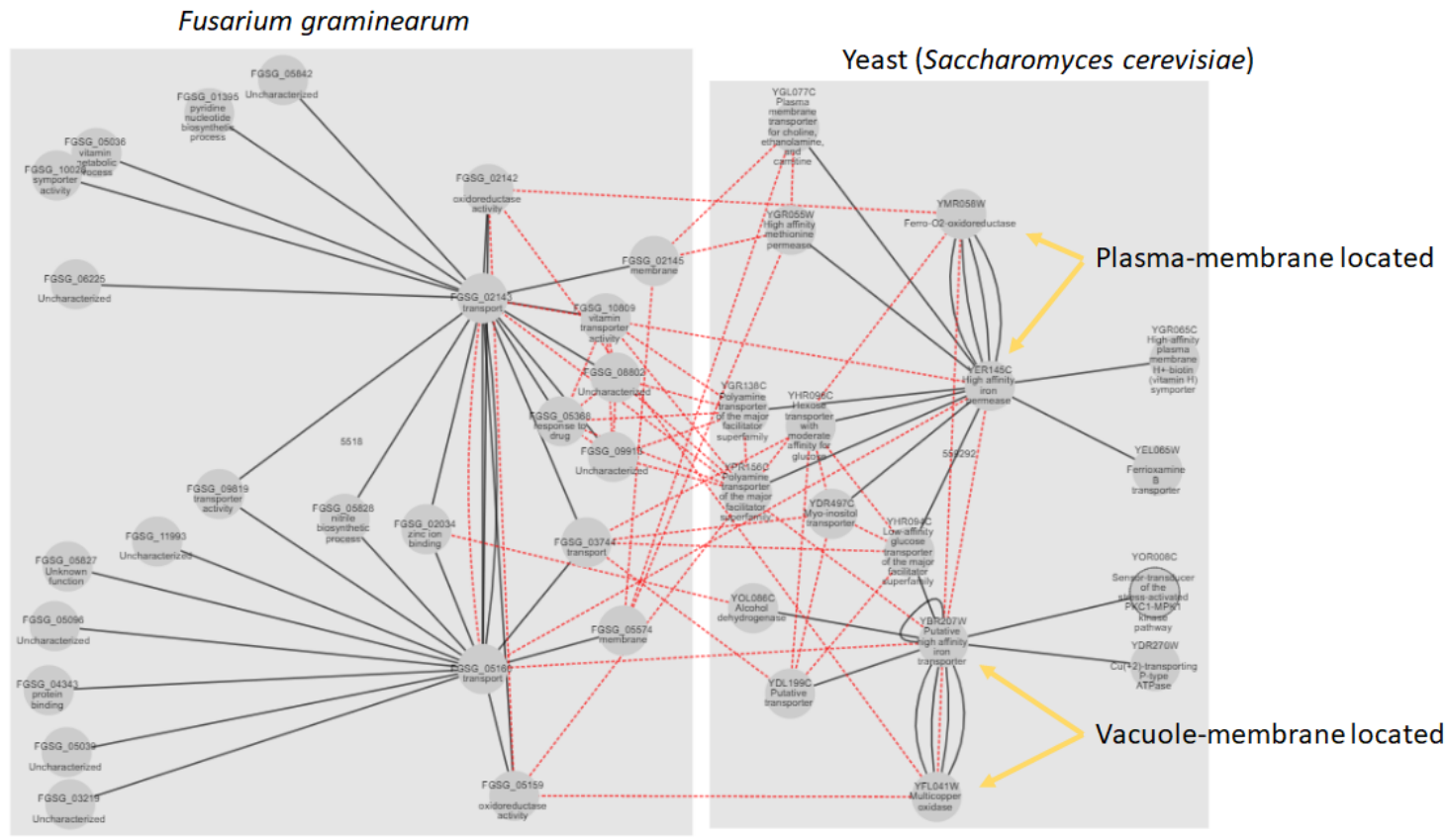

242 Fig 5. The alignment of the co-expression neighbourhoods from Yeast and F. graminearum,

243 Using FGSG_05160 as a bait, and searching for counterparts in Yeast with similar co-

244 expression patterns. Black edges represent co-expression edges, red edges represent

245 possible orthologs relationship. It can be seen that large parts of the network are mirrored

246 across the organisms, hinting towards the genes carrying out the same functionality.

\section{Conclusions and future directions}

248 In this study, we developed a system for handling biological omics data. We processed gene 249 expression data, as well as generated co-expression networks for 10 species. 
250 We established an interactive web interface omicsdb.org to provide access to the data as

251 well as the analysis platforms for the public. We plan to continue to improve the quality and

252 functionality of this database, by regularly updating with new publicly available data.

253 Currently the database contains networks for 4 pathogens and 6 references, however, there

254 is a growing need for understanding how plant pathogens work, to alleviate this need we will 255 include new species as when they become available. 


\section{External tools}

258 BLAST+ (Camacho et al., 2009) has been integrated to enable the users to easily find their 259 gene of interest, allowing the user to BLAST their bait sequence against our database. This 260 is practical in case they have identifiers not present in our database, or the user working on 261 species not yet included.

262

\section{Construction of gene co-expression data}

264 For the construction of co-expression database for plant pathogens, we selected 4 265 pathogenic species (Fusarium graminearum, Ustilago maydis, Blumeria graminis, 266 Neurospora crassa), based on the availability of gene expression data, as well as 6 267 reference species (Escherichia coli, Schizosaccharomyces pombe, Saccharomyces cerevisiae, Arabidopsis thaliana, Mus musculus, Homo sapiens).

269 Expression data were downloaded from Arrayexpress (Kolesnikov et al., 2015), and normalized using the following methods: For Agilent data, the R-package limma (Ritchie et al., 2015) were used . For Affymetrix, Affymetrix Powertools v. was used, running the RMA algorithm. For NimbleGen, DEVA V 1.02 (Roche) was used. RNA-Seq data were subjected to QC using FastQC (Andrews), reads were mapped using TopHat2 (Kim et al., 2013), counted using HTseq-count (Anders et al., 2015) and normalized using the VST algorithm implemented in DESeq2 (Love et al., 2014), run in R v. 3.4 (R Core Team, 2013).

Genome annotations were derived from Pombase for S. pombe (Wood et al., 2012), SGD for S. cerevisiae (Cherry et al., 1998), and Ensembl for the remaining species.

Transcription factor annotations were downloaded from (Liu et al., 2013), Enzyme Commision numbers were obtained from Uniprot (The Uniprot Consortium, 2019). Biological, chemical and genetic interactions were derived from BioGrid (Oughtred et al., 2019).

The Mutual Rank value of the weighted Pearson's correlation coefficient was used as the measure of co-expression, as described by (Obayashi et al., 2009). A threshold of 50 was applied. To make it comparable with protein interactions, the MR was normalized by taking $1 /$ MR ensuring that 1 was equivalent to the best value. Mutual Rank is calculated as

$$
\operatorname{MR}(A, B)=\sqrt{ }(\operatorname{Rank}(A \rightarrow B) \times \operatorname{Rank}(B \rightarrow A))
$$


289 With A and B represent genes. GFF files were stored in tabix format (Li, 2011) peptide and

290 DNA sequences were stored in faidx format implemented in Samtools (Li et al., 2009).

291 Functional Annotations

292 Protein domains including PFAM domains (Finn et al., 2014) and Panther (Mi et al., 2013)

293 were identified using InterProScan v. 5.29-68.0 (Hunter et al., 2009; Jones et al., 2014),

294 Ortholog groups were identified using EggNOG (Huerta-Cepas et al., 2016), which also

295 provided predicted GO terms. For E. coli, S. pombe, S. cerevisiae A. thaliana, M. musculus

296 and Homo Sapiens, experimentally validated GO terms could be downloaded from the

297 GeneOntology website (The Gene Ontology Consortium, 2019). The Gene Ontology website

298 also provides mappings from domains identified by InterProScan to their associated GO

299 terms. These maps were used to further improve the annotations.

300 Papers citing a given gene or gene family were retrieved from Uniprot (The Uniprot

301 Consortium, 2019).

302 For reference, Arabidopsis thaliana, Mus musculus and Homo sapiens were included,

303 annotations were derived similarly to the pathogens.

\section{Network alignment}

305 The network alignment uses the "@NOG” ortholog families, if you provide a bait gene, it will

306 query all other genes from this family, and compare how many orthologs are shared in the

307 1st-degree neighbourhood. To calculate how much of the network around two genes is

308 similar, the Jaccard index for the gene families shared across the networks is then

309 calculated following

310

$$
J(A, B)=\frac{|A \cap B|}{|A \cup B|}
$$

311 Where A and B represent the gene families in the network surrounding your bait gene and

312 the gene it is compared against respectively. The numerator is the intersection of gene

313 families, and denominator represents the union of all gene families in the 1st-degree

314 neighbourhood. 


\section{Ontology Enrichment}

316 Enrichment of Gene Ontology terms in sets of genes is calculated using the Hypergeometric

317 distribution function, implemented in Scipy (Virtanen et al., 2020). For the calculation of

318 background terms, following the true path rule, all ancestors of any given annotation are

319 considered.

320

Database implementation

321 The system runs on Linux v. 18.04 LTS Bionic Beaver. The web-service was implemented in

322 a Python-based web application framework, Flask v. 1.0.2, with SQLite and Neo4J 3.4

323 (www.neo4j.com) as the backend databases for the expression data and everything else

324 respectively. The website is being served using gunicorn and NGINX. The co-expressed

325 gene networks as well as the directed acyclic graphs for the GO terms were visualized using

326 Cytoscape.js v. 3.14 (Franz et al., 2016), gene expression plots were generated using dc.js

327 (https://dc-js.github.io/dc.js/). For the HTLM page layout, the bootstrap framework was used

328 (www.getbootstrap.com), and for general purpose usability features the Jquery.js library

329 (https://jquery.com) was used

330 BLAST+ (Camacho et al., 2009) was installed and a custom HTML interface was developed.

331 Icons were obtained from Font Awesome v. 5 (www.fontawesome.com).

332 Conflict of Interest

333 No conflicts are declared.

334 Author Contributions

$335 \mathrm{BOH}$ and $\mathrm{SO}$ designed the study, $\mathrm{BOH}$ developed the database and processed the data.

$336 \mathrm{BOH}$ and $\mathrm{SO}$ wrote the manuscript

\section{References}

338 Anders, S., Pyl, P. T., and Huber, W. (2015). HTSeq-a Python framework to work with 339 high-throughput sequencing data. Bioinformatics 31, 166-169.

340 doi:10.1093/bioinformatics/btu638.

341 Andorf, C. M., Cannon, E. K., Portwood, J. L., Gardiner, J. M., Harper, L. C., Schaeffer, M.

342 L., et al. (2016). MaizeGDB update: new tools, data and interface for the maize

343 model organism database. Nucleic Acids Res. 44, D1195-1201. 
doi:10.1093/nar/gkv1007.

Andrews, S. FastQC: a quality control tool for high throughput sequence data. Available at: http://www.bioinformatics.babraham.ac.uk/projects/fastqc [Accessed June 25, 2019].

Ashburner, M., Ball, C. A., Blake, J. A., Botstein, D., Butler, H., Cherry, J. M., et al. (2000). Gene ontology: tool for the unification of biology. The Gene Ontology Consortium. Nat. Genet. 25, 25-29. doi:10.1038/75556.

Askwith, C., Eide, D., Van Ho, A., Bernard, P. S., Li, L., Davis-Kaplan, S., et al. (1994). The FET3 gene of $S$. cerevisiae encodes a multicopper oxidase required for ferrous iron uptake. Cell 76, 403-410. doi:10.1016/0092-8674(94)90346-8.

Camacho, C., Coulouris, G., Avagyan, V., Ma, N., Papadopoulos, J., Bealer, K., et al. (2009). BLAST+: architecture and applications. BMC Bioinformatics 10, 421. doi:10.1186/1471-2105-10-421.

Cherry, J. M., Adler, C., Ball, C., Chervitz, S. A., Dwight, S. S., Hester, E. T., et al. (1998). SGD: Saccharomyces Genome Database. Nucleic Acids Res 26, 73-79. doi:10.1093/nar/26.1.73.

Cornish-Bowden, A. (2014). Current IUBMB recommendations on enzyme nomenclature and kinetics. Perspectives in Science 1, 74-87. doi:10.1016/j.pisc.2014.02.006.

Finn, R. D., Bateman, A., Clements, J., Coggill, P., Eberhardt, R. Y., Eddy, S. R., et al. (2014). Pfam: the protein families database. Nucleic Acids Res. 42, D222-230. doi:10.1093/nar/gkt1223.

Franz, M., Lopes, C. T., Huck, G., Dong, Y., Sumer, O., and Bader, G. D. (2016). Cytoscape.js: a graph theory library for visualisation and analysis. Bioinformatics 32 , 309-311. doi:10.1093/bioinformatics/btv557.

Galagan, J. E., Calvo, S. E., Borkovich, K. A., Selker, E. U., Read, N. D., Jaffe, D., et al. (2003). The genome sequence of the filamentous fungus Neurospora crassa. Nature 422, 859-868. doi:10.1038/nature01554.

Giaever, G., Chu, A. M., Ni, L., Connelly, C., Riles, L., Véronneau, S., et al. (2002). Functional profiling of the Saccharomyces cerevisiae genome. Nature 418, 387-391. doi:10.1038/nature00935.

Hansen, B. O., Vaid, N., Musialak-Lange, M., Janowski, M., and Mutwil, M. (2014). Elucidating gene function and function evolution through comparison of coexpression networks of plants. Front. Plant Sci. 5. doi:10.3389/fpls.2014.00394.

Have, C. T., and Jensen, L. J. (2013). Are graph databases ready for bioinformatics? Bioinformatics 29, 3107-3108. doi:10.1093/bioinformatics/btt549.

Huerta-Cepas, J., Szklarczyk, D., Forslund, K., Cook, H., Heller, D., Walter, M. C., et al. (2016). eggNOG 4.5: a hierarchical orthology framework with improved functional annotations for eukaryotic, prokaryotic and viral sequences. Nucleic Acids Res 44, 
381

382

383

384

385

386

387

388

389

390

391

392

393

394

395

396

397

398

399

400

401

402

403

404

405

406

407

408

409

410

411

412

413

414

415

416

417

D286-D293. doi:10.1093/nar/gkv1248.

Hunter, S., Apweiler, R., Attwood, T. K., Bairoch, A., Bateman, A., Binns, D., et al. (2009). InterPro: the integrative protein signature database. Nucleic Acids Res 37, D211D215. doi:10.1093/nar/gkn785.

Itkin, M., Heinig, U., Tzfadia, O., Bhide, A. J., Shinde, B., Cardenas, P. D., et al. (2013). Biosynthesis of antinutritional alkaloids in solanaceous crops is mediated by clustered genes. Science 341, 175-179. doi:10.1126/science.1240230.

Jones, P., Binns, D., Chang, H.-Y., Fraser, M., Li, W., McAnulla, C., et al. (2014). InterProScan 5: genome-scale protein function classification. Bioinformatics 30, 1236-1240. doi:10.1093/bioinformatics/btu031.

Kämper, J., Kahmann, R., Bölker, M., Ma, L.-J., Brefort, T., Saville, B. J., et al. (2006). Insights from the genome of the biotrophic fungal plant pathogen Ustilago maydis. Nature 444, 97-101. doi:10.1038/nature05248.

Kim, D., Pertea, G., Trapnell, C., Pimentel, H., Kelley, R., and Salzberg, S. L. (2013). TopHat2: accurate alignment of transcriptomes in the presence of insertions, deletions and gene fusions. Genome Biology 14, R36. doi:10.1186/gb-2013-14-4-r36.

King, R., Urban, M., Hammond-Kosack, M. C. U., Hassani-Pak, K., and Hammond-Kosack, K. E. (2015). The completed genome sequence of the pathogenic ascomycete fungus Fusarium graminearum. BMC Genomics 16, 544. doi:10.1186/s12864-0151756-1.

Kolesnikov, N., Hastings, E., Keays, M., Melnichuk, O., Tang, Y. A., Williams, E., et al. (2015). ArrayExpress update-simplifying data submissions. Nucleic Acids Res 43, D1113-D1116. doi:10.1093/nar/gku1057.

$\mathrm{Li}, \mathrm{H}$. (2011). Tabix: fast retrieval of sequence features from generic TAB-delimited files. Bioinformatics 27, 718-719. doi:10.1093/bioinformatics/btq671.

Li, H., Handsaker, B., Wysoker, A., Fennell, T., Ruan, J., Homer, N., et al. (2009). The Sequence Alignment/Map format and SAMtools. Bioinformatics 25, 2078-2079. doi:10.1093/bioinformatics/btp352.

Liu, X., Zhang, X., Tang, W.-H., Chen, L., and Zhao, X.-M. (2013). eFG: an electronic resource for Fusarium graminearum. Database (Oxford) 2013. doi:10.1093/database/bat042.

Love, M. I., Huber, W., and Anders, S. (2014). Moderated estimation of fold change and dispersion for RNA-seq data with DESeq2. Genome Biol 15. doi:10.1186/s13059014-0550-8.

Mi, H., Muruganujan, A., and Thomas, P. D. (2013). PANTHER in 2013: modeling the evolution of gene function, and other gene attributes, in the context of phylogenetic trees. Nucleic Acids Res. 41, D377-386. doi:10.1093/nar/gks1118. 
418 Movahedi, S., Bel, M. V., Heyndrickx, K. S., and Vandepoele, K. (2012). Comparative co-

419

420

421

422

423

424

425

426

427

428

429

430

431

432

433

434

435

436

437

438

439

440

441

442

443

444

445

446

447

448

449

450

451

452

453

454 expression analysis in plant biology. Plant, Cell \& Environment 35, 1787-1798. doi:10.1111/j.1365-3040.2012.02517.x.

Obayashi, T., Hayashi, S., Saeki, M., Ohta, H., and Kinoshita, K. (2009). ATTED-II provides coexpressed gene networks for Arabidopsis. Nucleic Acids Res. 37, D987-991. doi:10.1093/nar/gkn807.

Oughtred, R., Stark, C., Breitkreutz, B.-J., Rust, J., Boucher, L., Chang, C., et al. (2019). The BioGRID interaction database: 2019 update. Nucleic Acids Res 47, D529-D541. doi:10.1093/nar/gky1079.

Palankar, M. R., lamnitchi, A., Ripeanu, M., and Garfinkel, S. (2008). Amazon S3 for science grids: a viable solution? in Proceedings of the 2008 international workshop on Dataaware distributed computing DADC '08. (Boston, MA, USA: Association for Computing Machinery), 55-64. doi:10.1145/1383519.1383526.

Pedro, H., Maheswari, U., Urban, M., Irvine, A. G., Cuzick, A., McDowall, M. D., et al. (2016). PhytoPath: an integrative resource for plant pathogen genomics. Nucleic Acids Res 44, D688-D693. doi:10.1093/nar/gkv1052.

Ritchie, M. E., Phipson, B., Wu, D., Hu, Y., Law, C. W., Shi, W., et al. (2015). limma powers differential expression analyses for RNA-sequencing and microarray studies. Nucleic Acids Res 43, e47-e47. doi:10.1093/nar/gkv007.

Stearman, R., Yuan, D. S., Yamaguchi-Iwai, Y., Klausner, R. D., and Dancis, A. (1996). A Permease-Oxidase Complex Involved in High-Affinity Iron Uptake in Yeast. Science 271, 1552-1557. doi:10.1126/science.271.5255.1552.

Stuart, J. M., Segal, E., Koller, D., and Kim, S. K. (2003). A Gene-Coexpression Network for Global Discovery of Conserved Genetic Modules. Science 302, 249-255. doi:10.1126/science.1087447.

The Complete Genome Sequence of Escherichia coli K-12 | Science Available at: https://science.sciencemag.org/content/277/5331/1453 [Accessed August 7, 2019].

The Gene Ontology Consortium (2019). Gene Ontology Resource: 20 years and still GOing strong. Nucleic Acids Res 47, D330-D338. doi:10.1093/nar/gky1055.

The Uniprot Consortium (2019). UniProt: a worldwide hub of protein knowledge. Nucleic Acids Res 47, D506-D515. doi:10.1093/nar/gky1049.

Urbanowski, J. L., and Piper, R. C. (1999). The Iron Transporter Fth1p Forms a Complex with the Fet5 Iron Oxidase and Resides on the Vacuolar Membrane. J. Biol. Chem. 274, 38061-38070. doi:10.1074/jbc.274.53.38061.

Usadel, B., Obayashi, T., Mutwil, M., Giorgi, F. M., Bassel, G. W., Tanimoto, M., et al. (2009). Co-expression tools for plant biology: opportunities for hypothesis generation and caveats. Plant Cell Environ. 32, 1633-1651. doi:10.1111/j.1365- 
455

456

457

458

459

460

461

462

463

464

465

466

467

468

469

470

471

472

473

474

475

476

477

3040.2009.02040.x.

Virtanen, P., Gommers, R., Oliphant, T. E., Haberland, M., Reddy, T., Cournapeau, D., et al. (2020). SciPy 1.0: fundamental algorithms for scientific computing in Python. Nat Methods, 1-12. doi:10.1038/s41592-019-0686-2.

Vo, T. V., Das, J., Meyer, M. J., Cordero, N. A., Akturk, N., Wei, X., et al. (2016). A Proteome-wide Fission Yeast Interactome Reveals Network Evolution Principles from Yeasts to Human. Cell 164, 310-323. doi:10.1016/j.cell.2015.11.037.

Wicker, T., Oberhaensli, S., Parlange, F., Buchmann, J. P., Shatalina, M., Roffler, S., et al. (2013). The wheat powdery mildew genome shows the unique evolution of an obligate biotroph. Nature Genetics 45, 1092-1096. doi:10.1038/ng.2704.

Wolfe, C. J., Kohane, I. S., and Butte, A. J. (2005). Systematic survey reveals general applicability of "guilt-by-association" within gene coexpression networks. BMC Bioinformatics 6, 227. doi:10.1186/1471-2105-6-227.

Wood, V., Gwilliam, R., Rajandream, M.-A., Lyne, M., Lyne, R., Stewart, A., et al. (2002). The genome sequence of Schizosaccharomyces pombe. Nature 415, 871-880. doi:10.1038/nature724.

Wood, V., Harris, M. A., McDowall, M. D., Rutherford, K., Vaughan, B. W., Staines, D. M., et al. (2012). PomBase: a comprehensive online resource for fission yeast. Nucleic Acids Res 40, D695-D699. doi:10.1093/nar/gkr853.

Zhang, L., Zhang, D., Chen, Y., Ye, W., Lin, Q., Lu, G., et al. (2019). Magnaporthe oryzae CK2 Accumulates in Nuclei, Nucleoli, at Septal Pores and Forms a Large Ring Structure in Appressoria, and Is Involved in Rice Blast Pathogenesis. Front. Cell. Infect. Microbiol. 9. doi:10.3389/fcimb.2019.00113. 